\title{
Love in the time of coronavirus: training and service during COVID-19
}

\author{
Chen Seong Wong ${ }^{1,2}$, MBBS, MRCP, Woo Chiao $\underline{\text { Tay }^{1}}$, MBBS, Xing Fu $\underline{\text { Hap }}^{3}$, MBBS, MRCS, Faith Li-Ann $\underline{\text { Chia }}{ }^{4,5}$, MBBS, FRCP
}

\section{INTRODUCTION}

Reports of a viral pneumonia caused by a novel coronavirus, now termed coronavirus disease 2019 (COVID-19), first emerged in December 2019 in Wuhan, the capital city of Hubei province in China. As of 7 March 2020, there were 101,927 confirmed cases and 3,486 deaths reported worldwide. ${ }^{(1)}$ Singapore had 138 confirmed cases of COVID-19 as of 7 March 2020.(2) The Ministry of Health raised the public health response level to DORSCON (Disease Outbreak Response System Condition) Orange on 7 February 2020 in concert with a comprehensive suite of public health interventions aimed at containing the outbreak in Singapore, involving every level of the healthcare system. ${ }^{(3)}$ Singapore's response to the COVID-19 outbreak has been praised for its swiftness and focus on contact tracing, containment and proactive surveillance. ${ }^{(4,5)}$

The epicentre for the national response has been the National Centre for Infectious Diseases (NCID), with manpower support from Tan Tock Seng Hospital (TTSH), on the Novena campus of the National Healthcare Group (NHG). NCID has seen the majority of the patients screened, diagnosed and managed for COVID-19. By January 2020, TTSH and NCID had pre-emptively shifted operations into outbreak response mode, deploying resources and manpower to outbreak wards and the Screening Centre at NCID, with a concomitant scale-down of routine and elective services. This further intensified during DORSCON Orange.

NHG is home to 27 residency programmes, with a total of 553 residents and more than 3,000 clinical faculty forming a large part of the clinical personnel within its institutions. When the call first came for residents to contribute to the institutional response, they did so willingly and unhesitatingly, with many volunteering to serve in outbreak response roles. Following its designation as the national response centre for SARS (severe acute respiratory syndrome) in 2003 and subsequent infectious disease outbreaks thereafter, TTSH and its staff have come to consider outbreak response to be an intrinsic part of their roles in Singapore healthcare - perhaps even as an essential 'national service'.(6)

The COVID-19 outbreak, and NHG's response to it, has had wide-ranging effects on its residents, especially the interruptions to training that have ensued as well as ever-growing disruptions to daily practice, career and personal life. At the same time, residents and other junior clinicians are a critical component of the COVID-19 response, and their contributions to service will be required as long as the outbreak continues. With the outbreak showing no signs of slowing down in the region and internationally, we must be prepared that outbreak deployment and its attendant disruptions will continue well into 2020, and care must be taken to ensure that any detrimental effects are minimised for a generation of residents. ${ }^{(7)}$

From 2 March to 6 March 2020, the NHG Residency Office invited residents to take part in a quality improvement exercise to find out more about their experiences during their deployment to outbreak wards, clinics and the Screening Centre, all of which are sited at the NCID. The aim of this survey was to gather feedback about clinical work in outbreak areas, elucidate challenges residents might be facing and elicit suggestions for improvements. Participation in the survey was entirely voluntary, and responses were anonymous. Only residents who had been deployed to NCID between 17 February and 6 March were invited to respond. As this was a quality improvement exercise conducted by NHG Residency and its Resident Council, ethics approval was not required.

For this paper, we analysed responses to questions pertaining to the challenges faced by residents as a result of the interruptions to training posed by the ongoing COVID-19 outbreak. There were a total of 61 responses to the survey. Of these, $40 \%$ were from medical specialties, $40 \%$ from surgical specialties, $11 \%$ from family medicine (FM) and $9 \%$ from other specialties. These residents represented a wide range of clinical experience, from Postgraduate Year (PGY) 2 to PGY 10.

\section{SERVICE VERSUS TRAINING: A CHALLENGING BALANCE}

The deployment of residents from their positions of everyday work to handle outbreak requirements has included doctors from the Division of Medicine contributing to inpatient ward management at NCID, and those from the Division of Surgery contributing to shifts at the NCID Screening Centre, which serves as a screening facility for patients suspected of COVID-19. This deployment and reassignment has interrupted training, as residents are no longer working and learning within the subspecialty of their respective programmes and both didactic and clinical teaching have been stopped in order to meet service demands. This is especially significant in training programmes where skill and competence acquisition is linked to clinical caseload. In addition, due to the restrictions on staff movement and gathering, as well

${ }^{1}$ Department of Infectious Diseases, Tan Tock Seng Hospital, ${ }^{2}$ National Centre for Infectious Diseases, ${ }^{3}$ Department of Orthopaedic Surgery, Tan Tock Seng Hospital, ${ }^{4}$ National Healthcare Group Residency, ${ }^{5}$ Department of Rheumatology, Allergy and Immunology, Tan Tock Seng Hospital, Singapore

Correspondence: Dr Wong Chen Seong, Consultant, Department of Infectious Diseases, Tan Tock Seng Hospital, 11 Jalan Tan Tock Seng, Singapore 308433. chen_seong_wong@ttsh.com.sg 
as business continuity plans requiring splitting departments and teams to reduce the risk of inadvertent cross-contamination, many training and teaching programmes have been affected, including lectures, courses and workshops (including those required for essential credentials such as Basic and Advanced Cardiac Life Support) and cross-institutional clinical rotations. Moreover, many postgraduate professional examinations (including membership examinations for the various Royal Colleges) and their associated preparatory courses have been cancelled or postponed.

When asked whether the current outbreak had adversely affected their training and/or career, $61 \%$ of the respondents agreed or strongly agreed, with 35\% strongly agreeing, while $42 \%$ of all respondents either agreed or strongly agreed that the outbreak would adversely affect their ability to complete training and/or become a good specialist. $45 \%$ of all respondents agreed or strongly agreed that the outbreak had made them anxious about their training and/or career.

Residency programmes and institutions must be fully cognisant of the disruptions to training and the resultant anxieties faced by trainees, and take categorical action to ameliorate these. Personnel rosters and work schedules for deployed residents should be overseen with care to ensure trainees do not spend excessive amounts of time away from core work that is specific to their training. Alternative pedagogical strategies in times of outbreak, such as the use of technology through e-learning and simulations, have already been proposed and introduced in the local context and should be utilised now. ${ }^{(8)}$ In response to the cessation of in-person training events such as tutorials for examination preparation, journal clubs, and departmental and hospital-level teaching, NHG Residency has adopted the use of teleconferencing technology (e.g. Zoom) to facilitate teaching of core subjects. Regarding the restriction of movement across institutions, interrupting core cross-institutional and cross-cluster clinical rotations, NHG Residency has made arrangements to facilitate residents to make up required rotations at a later date and, as far as possible, will do its best to ensure alternate means of achieving and documenting competencies to allow affected residents to progress. Despite these remedial actions, some residents still may not meet the minimum criteria to progress or complete training (primarily due to an inability to accrue an adequate case mix or log sufficient hands-on procedural experience). Efforts to monitor the situation and develop long-term solutions are ongoing and must continue.

\section{CHALLENGES TO PRACTICE AND SERVICE}

Outbreak response clinical duties require the adoption of strict infection control as well as personal protection equipment (PPE) and practices to minimise the risk of infection. Many of these are rarely used in everyday practice and may be cumbersome, uncomfortable and stressful to use. Outbreak wards and clinics may also be unfamiliar to residents who do not usually practise in such settings, and there is evidence to suggest that working in such an environment is particularly challenging. ${ }^{(9,10)}$

Of the respondents in our survey who had been deployed to outbreak work at NCID, 55\% responded that this work was in an area beyond their usual scope of practice. When asked what they found challenging about their deployed roles, 32\% cited the workplace environment, $23 \%$ cited the unfamiliar work scope, $21 \%$ cited high caseloads and inadequate manpower, and 14\% cited the use of PPE. Despite this, when questioned if they felt equipped and/or prepared to perform their deployed roles, $49 \%$ and $18 \%$ of respondents agreed and strongly agreed, respectively. When asked about problems faced outside of the workplace, $36 \%$ of respondents cited disruption of leave and travel plans, while $29 \%$ and $25 \%$ cited family and interpersonal relationship challenges, respectively.

We must continue to do our utmost to ensure that deployed residents are adequately prepared and equipped to do the work asked of them. All residents must be trained in the use of PPE, and care must also be taken to ensure that they have confidence in the protection that it provides. Time and effort should be taken to properly orientate and train junior doctors who may be unfamiliar with the unique work that an outbreak response demands, especially one that requires strict infection prevention measures and specialised infectious disease expertise. Much of this training must be 'just-in-time', on-the-job training, but its importance in ensuring the safety and security of residents cannot be overstated.

\section{STRESS AND BURNOUT}

Lastly, human resources policies enacted during this time, such as a freeze on annual and training leave, cancellation of overseas travel and mandatory split team assignments (to minimise the risk of inadvertent transmission of infection within departments and/or teams), have had an effect on the conduct of training as well as morale and emotional well-being. When asked to rate the level of stress experienced during their deployment on a scale of 0 (no stress at all) to 10 (extreme levels of stress), the mean level was 4.7, with medical, surgical and FM residents having mean stress levels of 5.5, 3.0 and 8.3, respectively. When asked to rate their level of resilience on a similar scale of $0-10$, the mean level was 7.52 across all residency programme types.

Residency programmes and institutions must recognise that residents, and in fact all junior doctors, face considerable stress and risk burnout when committed to outbreak response at such an early stage in their careers, despite reporting high levels of self-rated resilience. Particular attention may need to be paid to residents in the FM residency programme, who reported higher stress levels. This may be due to the intrinsic nature of FM training involving shorter postings in both acute hospitals and polyclinics, which were more severely disrupted in the current situation. Such stressors are not unique to COVID-19 and have been previously studied. They include fear of exposure to infection and the subsequent risk to families and loved ones, as well as concerns that their own education and careers are being compromised. ${ }^{(11)}$ Efforts should be taken to ensure that the emotional and psychological welfare of all staff, including junior staff and residents, are actively monitored and holistic programmes put in place to protect them. These may include the nomination of key departmental and institutional officers in charge of staff welfare, maintaining open and bidirectional lines 
of communication in disseminating information and changes in workplace policies, and reviewing staffing policies regularly to ensure fair and equitable personnel deployment. Efforts to prioritise staff wellness and promote resilience are important, and have been shown to enhance the professionalism of healthcare workers and create safer, happier work environments. ${ }^{(12,13)}$

\section{SMALL VICTORIES IN A LONG WAR}

The COVID-19 outbreak, and NHG's response to it, has had several positive effects. The call to contribute to institutional and national need has been met steadily and enthusiastically by residents in NHG, and appreciation and recognition for this has come from within the institution as well as members of the public. Residents have risen to the occasion and not only provided clinical manpower but also played important roles in supporting each other and their respective programmes. Their involvement in the various facets of this outbreak is an invaluable real-world education in crisis management on a large scale. It has also afforded an opportunity for residents from different specialties and disciplines that may exist in silos in 'peacetime' to come together in a time of great need. These small victories are well worth building on in the short and long term.

It is imperative for leaders in healthcare and medical education alike to ensure that our residents - who have unflinchingly answered the call to arms and will be asked to continue to do so for the duration of this pandemic - are cared for, trained and mentored in a way that befits the next generation of our nation's healthcare professionals.

\section{ACKNOWLEDGMENTS}

The authors would like to thank the residents who responded to the survey for their participation, as well as all medical officers and junior/senior residents for their continued contributions to the ongoing COVID-19 outbreak response.

\section{REFERENCES}

1. World Health Organization. Coronavirus disease 2019 (COVID-19) Situation Report - 47. Available at: https://www.who.int/docs/default-source/coronaviruse/ situation-reports/20200307-sitrep-47-covid-19.pdf?sfvrsn=27c364a4_4. Accessed March 8, 2020.

2. Ministry of Health, Singapore. Eight more cases discharged, eight new cases of COVID-19 infection confirmed. Available at: https://www.moh.gov.sg/newshighlights/details/eight-more-cases-discharged-eight-more-cases-of-covid-19infection-confirmed Accessed March 8, 2020.

3. Wong JEL, Leo YS, Tan CC. COVID-19 in Singapore-current experience: critical global issues that require attention and action. JAMA 2020 Feb 20. https://doi. org/10.1001/jama.2020.2467. [Epub ahead of print]

4. WHO 'very impressed' with Singapore's COVID-19 response. In: Channel NewsAsia [online]. Available at: https://www.channelnewsasia.com/news/ singapore/singapore-very-impressed-covid-19-coronavirus-response-whochief-12448486. Accessed March 8, 2020.

5. Hsu LY, Chia PY, Vasoo S. A midpoint perspective on the COVID-19 pandemic. Singapore Med J 2020; 61:381-3.

6. Chee YC. Heroes and heroines of the war on SARS. Singapore Med J 2003; $44: 221$.

7. World Health Organization. WHO statement on cases of COVID-19 surpassing 100,000. Available at: https://www.who.int/news-room/detail/07-03-2020who-statement-on-cases-of-covid-19-surpassing-100-000. Accessed March 8, 2020.

8. Lim EC, Oh VM, Koh DR, Seet RC. The challenges of "continuing medical education" in a pandemic era. Ann Acad Med Singapore 2009; 38: 724-6.

9. Khee KS, Lee LB, Chai OT, et al. The psychological impact of SARS on health care providers. Crit Care Shock 2004; 7:99-106.

10. KY Tham, YH Tan, OH Loh, WL Tan, HK Tang. Psychological morbidity among emergency department doctors and nurses after the SARS outbreak. Hong Kong J Emerg Med 2005; 12:215-23.

11. Rambaldini G, Wilson K, Rath D, et al. The impact of severe acute respiratory syndrome on medical house staff: a qualitative study. J Gen Intern Med 2005; 20:381-5.

12. Straus SE, Kumanan W, Rambaldini G, et al. Severe acute respiratory syndrome and its impact on professionalism: qualitative study of physicians' behavior during an emerging healthcare crisis. BMJ 2004; 329:83.

13. Aiello A, Khayeri MY, Raja S, et al. Resilience training for hospital workers in anticipation of an influenza pandemic. J Contin Educ Health Prof 2011; 31:15-20. 